\title{
Health care professionals' knowledge about percutaneous gastrostomy feeding. How well are we doing?
}

\author{
S. Thomas ${ }^{1}$ and F. C. Leslie ${ }^{2}$ \\ ${ }^{1}$ School of Medicine, University of Keele and ${ }^{2}$ Department of Gastroenterology, University Hospital of North Staffordshire, \\ Stoke-on-Trent, Staffordshire ST4 $6 Q G$
}

There is a reasonable body of evidence to support the use of percutaneous gastrostomy (PEG) feeding for stroke patients, but there is no evidence to support this practice in advanced dementia. A previous study published in 2001, showed that $34 \%$ of UK consultants would refer a patient with dementia for a PEG ${ }^{(1)}$ and a US study reported that $76 \%$ of physicians thought that PEG feeding prevented aspiration pneumonia in advanced dementia patients ${ }^{(2)}$. However, only $18 \%$ of dietitians said they would want a PEG for themselves in the event of advanced dementia ${ }^{(3)}$. In the light of the Mental Capacity Act it is vital that health care professionals are able to make evidence based decisions and advise patients and families appropriately.

We were keen to explore health care professionals' knowledge about PEG feeding. We were also keen to identify if teaching had any influence on knowledge. A simple questionnaire was given to a range of health care professionals in 4 hospitals across the Midlands and to an expert group (Midlands Gastroenterology Meeting), as part of a medical student project. Participants were asked about two scenarios of dysphagic stroke and advanced dementia. Questions asked were 'Would you refer the patient for a PEG, will it improve their quality of life or life expectancy and will it prevent aspiration pneumonia?' They were also asked if they had received any teaching and how much relevant experience they had. Statistics were analysed using SPSS.

556 of 714 questionnaires were collected $(78 \%)$ from hospital doctors of all levels and GPs, medical students, nurses and dietitians. More respondents answered questions about dysphagic stroke correctly compared to dementia. $89 \%$ of consultants would refer a CVA patient for a PEG compared to $68 \%$ of $\mathrm{F} 1 / \mathrm{F} 2$ s. Only $10 \%$ of hospital consultants would refer the dementia patient for a PEG compared to $31 \%$ of GPs, $37 \%$ of F1/F2s, $36 \%$ of medical students and $45 \%$ of nurses. Only $29 \%$ of all respondents felt that PEG would prevent aspiration pneumonia in the dementia patient (although nurses opinion was split $45 \% v .45 \%$ ). $20 \%$ of respondents felt PEG would improve life expectancy. Dietitians as a group were most likely to answer questions correctly. $64 \%$ of respondents had experience with similar patients, including $77 \%$ of GPs and $69 \%$ of foundation doctors. There was no difference across the four hospitals.

Teaching significantly influenced answers for the dementia scenario $(P=0.001)$. Only $29 \%$ had received teaching and only $4 \%$ of GPs had attended relevant teaching. Experts were more likely to answer correctly $(P=0.004)$ and physicians more than surgeons $(P=0.03)$. Many respondents commented that they would welcome more teaching.

Despite improvements in consultants' knowledge compared with previous studies, there are still significant gaps in knowledge. Training improves knowledge and this study suggests that more teaching should be planned, particularly aimed at nurses, medical students, junior doctors and GPs particularly as the GPs are often the professionals involved in family discussions on such difficult ethical issues.

1. Sinha U et al. (2001) Arch Gerontol Geriatr 32, 113-118.

2. Shega J \& Hougham G (2003) J Palliat Med 885-893.

3. Healy S \& Mcnamara E (2002) J Hum Nutr Diet 15, 445-453. 